\title{
Synthesis and charge-transfer complexation studies on bis(aminomethyl) $m$-terphenyl based bis-oxycyclophanes with intra-annular amide functionality
}

\author{
Perumal Rajakumar, ${ }^{\mathrm{a}, *}$ and Ramar Padmanabhan ${ }^{\mathrm{b}}$ \\ ${ }^{a}$ Department of Organic Chemistry, University of Madras, Guindy Campus, \\ Chennai - 600 025, India \\ ${ }^{b}$ Research and Development Centre, Orchid Chemicals and Pharmaceuticals Ltd., \\ Sozhanganallur, Chennai-600 119, India \\ E-mail: perumalrajakumar@gmail.com
}

\begin{abstract}
Bis(aminomethyl) $m$-terphenyl based bis-oxy cyclophanes with amide group as intra-annular functionality were synthesized and characterized from spectral and analytical data. All the cyclophanes form 1:1 charge-transfer (CT) complex with TCNQ.
\end{abstract}

Keywords: Cyclophane amides, bis-oxy amides, charge transfer complex, TCNQ

\section{Introduction}

Synthesis of architecturally novel supramolecules in the context of designing simple models for studying supramolecular interactions stimulates the synthetic chemist to modify the molecular structures. Introduction of amide, ester functionality ${ }^{1-4}$ at the annular ring of cyclophanes would make them as models of protein-metal binding sites in biological systems. ${ }^{5-7}$ Manganese-oxo complexes of porphyrin ligands ${ }^{8,9}$ are reactive intermediates in O-atom transfer process. Cyclophanes containing both ether and amide functionality at the intra-annular ring system show selectivity in the complexation of metal ions. ${ }^{10}$ The biphenyl ${ }^{11}$ based cyclic amides have been reported for anion complexation. ${ }^{12}$ Supramolecular amides were used as molecular receptors ${ }^{13}$ and in molecular recognition ${ }^{14,15}$ of biologically interacting substrates including anti-HIV active macrocyclic amides. ${ }^{16}$ Synthesis of amide based supramolecular systems has been reported in the literature. ${ }^{17-20}$ The most important aspect of supramolecular chemistry is the host-guest complexation. Interactions between electron donors and complementary electron acceptor groups in cyclophanes can form intramolecular charge-transfer (CT) complexes and can exhibit self complementary properties in addition to $\pi-\pi$ interaction. ${ }^{21-23}$ Hence it is of interest to synthesize 
bis-oxy cyclophane diamides $\mathbf{1 - 5}$ and study their CT complexation properties with 7,7,8,8tetracyanoquinodimethane (TCNQ), tetracyanoethylene (TCNE) and paraquat (PQT).

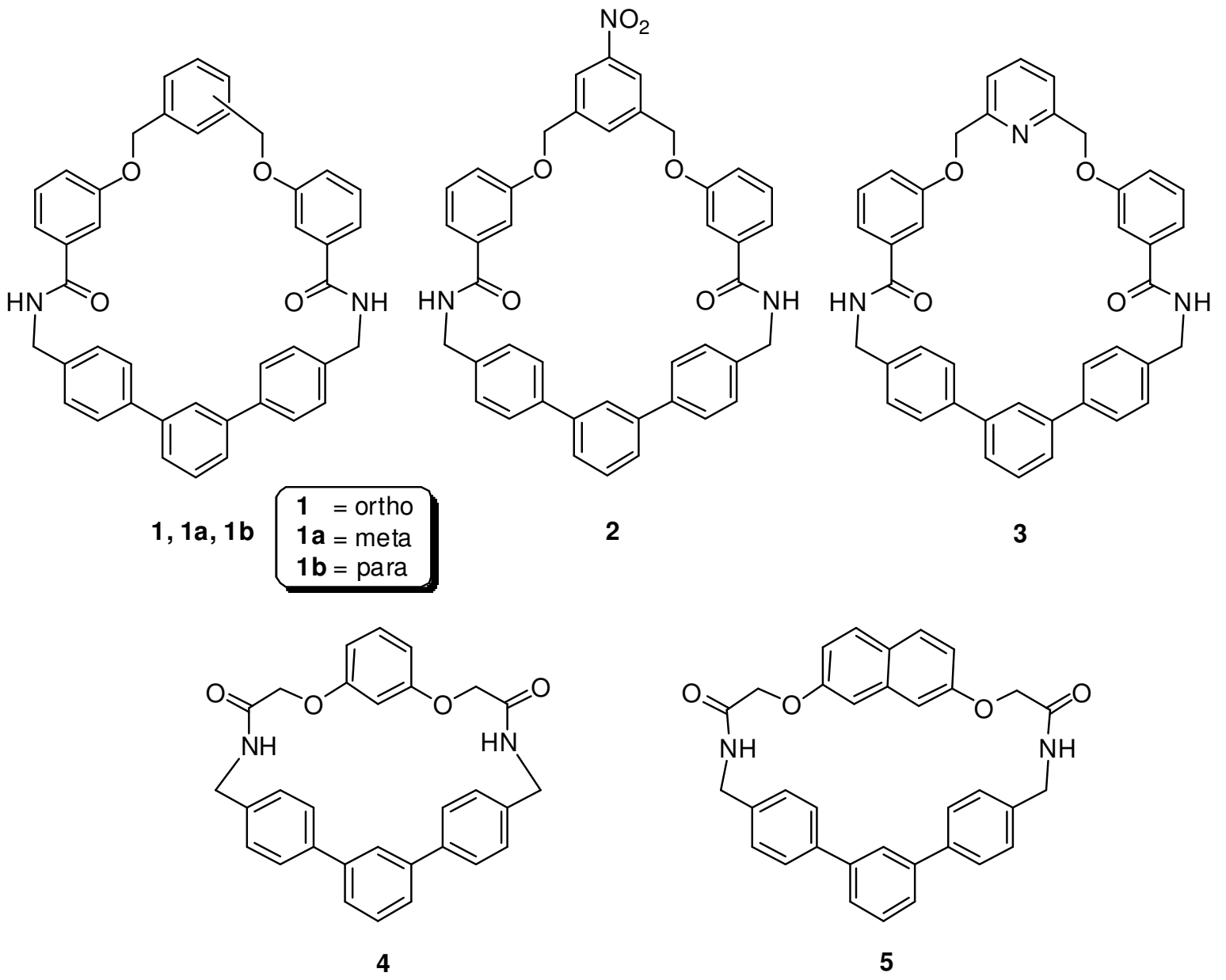

Figure 1. Structures of cyclophane amides 1-5.

\section{Results and Discussion}

Seven different bis-oxy cyclophane amides 1-5 shown in Figure 1 were synthesized from a novel bis(aminomethyl) $m$-terphenyl 22. Reaction of 1.0 equiv. of bis(bromomethyl) $m$-terphenyl $\mathbf{2 1}^{24}$ with 2.2 equiv. of hexamine ${ }^{25}$ in chloroform at reflux resulted in the formation of hexammonium salt. Hydrolysis of hexammonium salt with hydrochloric acid in $\mathrm{EtOH}-\mathrm{H}_{2} \mathrm{O}$ mixture at reflux afforded diamine $\mathbf{2 2}$ in about $90 \%$ yield (Scheme 1). The structure of diamine $\mathbf{2 2}$ was confirmed from the spectral data. The ${ }^{1} \mathrm{H}$ NMR spectrum of diamine 22 displayed the $N$-methylene protons as a singlet at $\delta$ 3.93. The rest of the aromatic protons appeared in the region $\delta$ 7.26-7.79.

Reaction of 1.0 equiv. of $o, m, p$-xylylene dibromide, $m$-nitro xylylene dibromide and 2,6bis(bromomethyl) pyridine with 2.3 equiv. of ethyl $m$-hydroxybenzoate in the presence of 3.1 
equiv. potassium carbonate in DMF at room temperature resulted in the formation of the corresponding bis-oxy esters 6, 6a, 6b, 7, and 8 respectively in about $85-95 \%$ yields after purification by column chromatography. Similarly reaction of 1.0 equiv. of resorcinol and

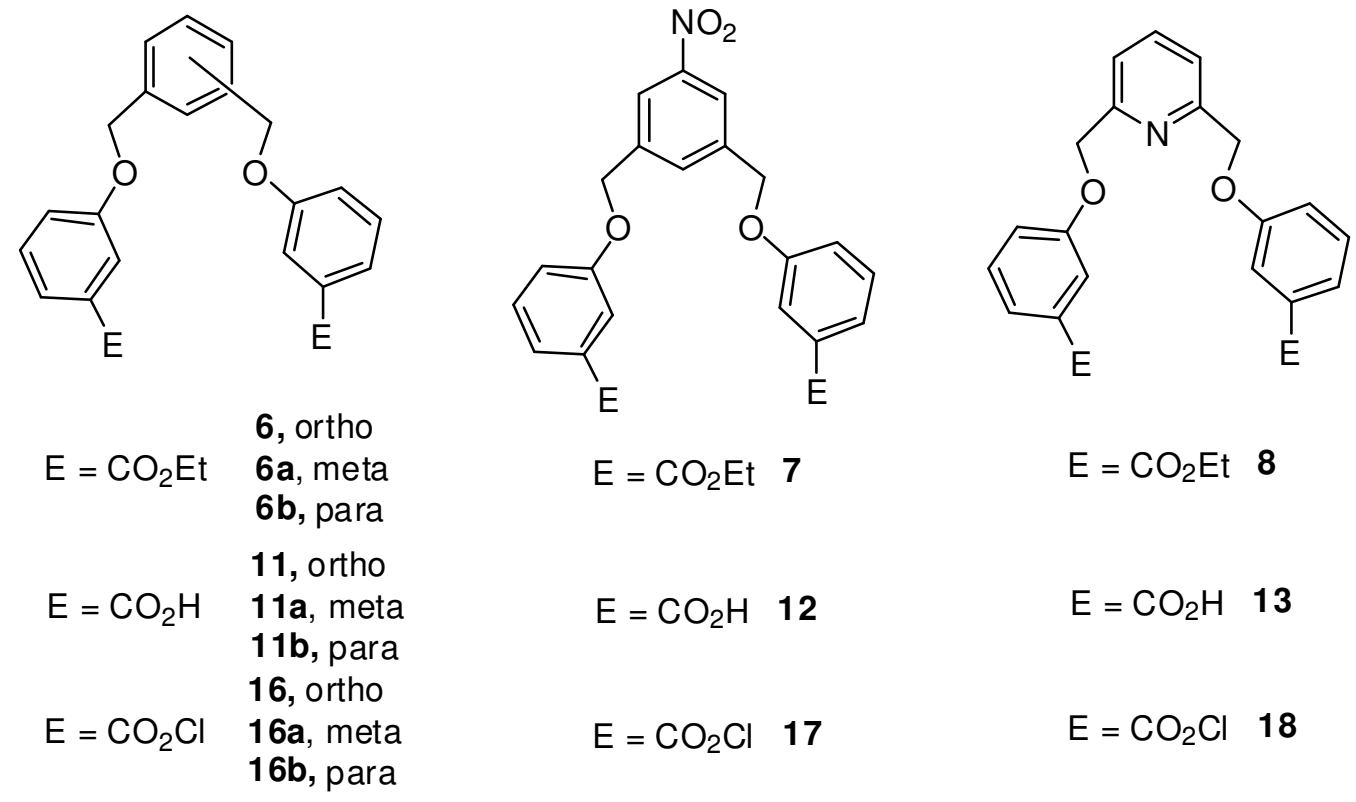

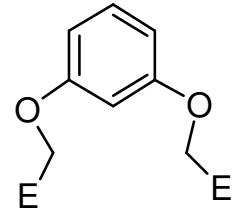

$\mathrm{E}=\mathrm{CO}_{2} \mathrm{Et} \quad 9$

$\mathrm{E}=\mathrm{CO}_{2} \mathrm{H} \quad 14$

$\mathrm{E}=\mathrm{CO}_{2} \mathrm{Cl} 19$<smiles>FCOc1ccc2ccc(OCF)cc2c1</smiles>

$\mathrm{E}=\mathrm{CO}_{2} \mathrm{Et} \quad 10$

$\mathrm{E}=\mathrm{CO}_{2} \mathrm{H} \quad 15$

$\mathrm{E}=\mathrm{CO}_{2} \mathrm{Cl} 20$

Figure 2. Structures of dicarboxylic acid ester 6-10, dicarboxylic acid 11-15 and dicarboxylic acid chloride 16-20.

2,8-naphthalenediol with 2.2 equiv. of ethyl bromoacetate in the presence of 3.1 equiv. potassium carbonate in acetonitrile in the presence of catalytic amount of KI at reflux for $8 \mathrm{~h}$ resulted in the formation of bis-oxy esters $\mathbf{9}$, and $\mathbf{1 0}$ respectively in about $80-95 \%$ yields after purification by column chromatography. The structure of bis-oxy esters $6,6 \mathbf{a}, 6 \mathbf{b}, 7,8,9$ and 10 was confirmed from spectral and analytical data. Hydrolysis of bis-oxy esters with potassium hydroxide in aqueous ethanol at reflux followed by acidification with $6 \mathrm{M} \mathrm{HCl}$ afforded the corresponding bis-oxy acids 11, 11a, 11b, 12, 13, 14 and 15 in about 90-95\% yields. The structure of bis-oxy acids 11, 11a, 11b, 12, 13, 14 and 15 was confirmed from spectral and 
analytical data. The corresponding bis-oxy acid chlorides 16, 16a, 16b, 17, 18, 19 and 20 were prepared by reacting the corresponding bis-oxy acids with thionyl chloride in the presence of TEA in DCM (Figure 2).
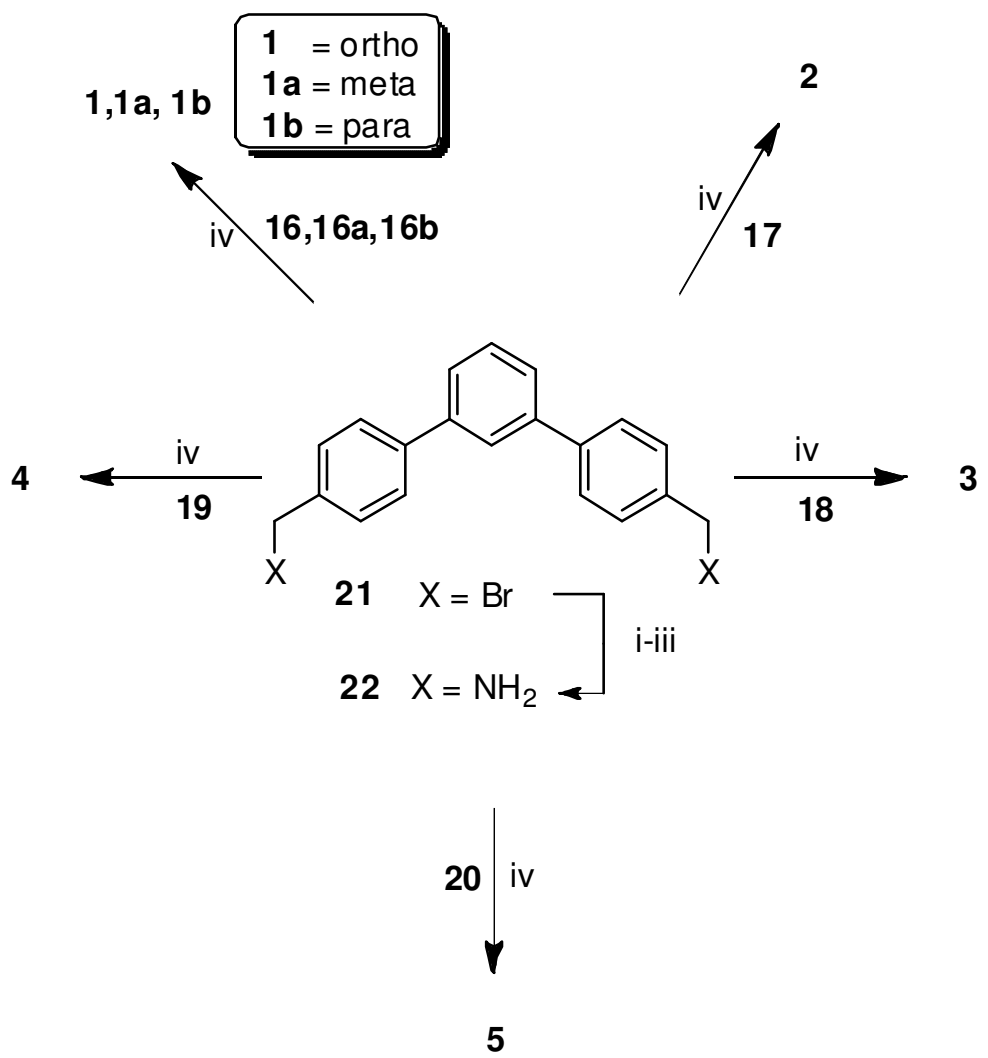

Scheme 1. Reagents and conditions: (i) Hexamine, $\mathrm{CHCl}_{3}, \mathrm{rt}, 12 \mathrm{~h}$; (ii) Conc. $\mathrm{HCl}, \mathrm{EtOH}-\mathrm{H}_{2} \mathrm{O}$, reflux, 3 h; (iii) $\mathrm{NaOH}-\mathrm{H}_{2} \mathrm{O}$, rt, 22 (90\%); (iv) TEA, DCM, rt, 24 h, 1 (35\%), 1a (40\%), 1b (52\%), 2 (42\%), 3 (43\%), 4 (30\%) and 5 (40\%).

In order to test the synthetic utility of diamine $\mathbf{2 2}$ for the synthesis of bis-oxy cyclophane diamide, 1.0 equiv of diamine 22 was coupled with 1.1 equiv of diacid chloride $\mathbf{1 6}, \mathbf{1 6 a}, \mathbf{1 6} \mathbf{b}, \mathbf{1 7}$, 18, 19 and 20 in the presence of triethylamine in dry DCM at room temperature under high dilution conditions. The reaction afforded the bis-oxy cyclophane diamides 1, 1a, 1b, 2, 3, 4 and 5 in $35,40,52,42,43,30$ and $40 \%$ yields respectively, after purification by column chromatography (Scheme 1). The ${ }^{1} \mathrm{H}$ NMR spectrum of cyclophane amide 1a displayed a doublet for the $N$-methylene protons at $\delta 4.51$, a singlet for $O$-methylene protons at $\delta 5.13$ and $\mathrm{NH}$ protons as a triplet at $\delta 9.03$. The rest of the aromatic protons appeared between $\delta 7.22$ and 7.73. In the ${ }^{13} \mathrm{C}$ NMR spectrum of cyclophane amide 1a, the $N$-methylene carbon appeared at $\delta$ 42.2, $O$-methylene carbons at $\delta 69.6$ and carbonyl carbons at $\delta$ 166.7. The FT-IR spectrum showed the carbonyl stretching frequency at $1639 \mathrm{~cm}^{-1}$ for the cyclophane amide 1a. Similarly 
the structure of the cyclophane amides $1, \mathbf{1 b}, \mathbf{2}, \mathbf{3}, \mathbf{4}$ and $\mathbf{5}$ has been confirmed from the spectral and analytical data.

\section{Determination of association constant (Ka) by Benesi-Hildebrand method}

The presence of ether and amide functionality in cyclophanes 1-5 can make them good molecular receptors for electron deficient guest molecules like TCNQ, TCNE and PQT. Cyclophane amides 1, 1a, 1b, 2, 3, 4 and 5 exhibited the formation of charge transfer complexes with TCNQ. However, complexation studies of compounds $\mathbf{1}, \mathbf{1 a}, \mathbf{1 b}, \mathbf{2}, \mathbf{3}, \mathbf{4}$ and $\mathbf{5}$ with TCNE and PQT were

Table 1. $\lambda_{\max }$ for cyclophane amides and TCNQ complex of cyclophane amides $\mathbf{1}, \mathbf{1 a}, \mathbf{1 b}, \mathbf{2}, \mathbf{3}$, 4 and 5

\begin{tabular}{cccc}
\hline $\begin{array}{c}\text { Conc. of guest, } \\
{[\mathrm{X}](\mathrm{M})}\end{array}$ & $\begin{array}{c}\text { Absorbance, } \\
\mathrm{A}\end{array}$ & {$[\mathrm{Y}] / \mathrm{A}(\mathrm{M})$} & $1 /[\mathrm{X}]\left(M^{-1}\right)$ \\
\hline $4.9 \times 10^{-6}$ & 0.032 & 0.000591 & 204081 \\
$9.8 \times 10^{-6}$ & 0.051 & 0.000371 & 102040 \\
$14.7 \times 10^{-6}$ & 0.064 & 0.000295 & 68027 \\
$19.6 \times 10^{-6}$ & 0.073 & 0.000259 & 51020 \\
$24.5 \times 10^{-6}$ & 0.080 & 0.000236 & 40816 \\
$29.4 \times 10^{-6}$ & 0.088 & 0.000215 & 34013 \\
\hline
\end{tabular}

not successful. However, cyclophanes 1, 1a, 1b, 2, 3, $\mathbf{4}$ and $\mathbf{5}$ show UV-Vis absorption maxima at 256.0, 256.0, 256.5, 257.0 and $256.0 \mathrm{~nm}$ respectively and the acceptor TCNQ shows an absorption maximum at $393.0 \mathrm{~nm}$. Cyclophanes 1, 1a, 1b, 2, 3, 4 and $\mathbf{5}$ form a charge-transfer

Table 2. Complexation of TCNQ with cyclophane amides $\mathbf{1}, \mathbf{1 a}, \mathbf{1 b}, \mathbf{2}, \mathbf{3}, \mathbf{4}$ and $\mathbf{5}$

\begin{tabular}{cccc}
\hline $\begin{array}{c}\text { Cyclophane } \\
\text { amide }\end{array}$ & $\mathrm{Ka}\left(\mathrm{mol}^{-1} \mathrm{dm}^{3}\right)$ & $\varepsilon\left[\mathrm{M}^{-1} \mathrm{~cm}^{-1}\right]$ & $\mathrm{r}$ \\
\hline $\mathbf{1}$ & $6.52 \times 10^{4}$ & $4.36 \times 10^{3}$ & 0.9999 \\
$\mathbf{1 a}$ & $6.65 \times 10^{4}$ & $6.87 \times 10^{3}$ & 0.9998 \\
$\mathbf{1 b}$ & $8.75 \times 10^{4}$ & $4.04 \times 10^{3}$ & 0.9994 \\
$\mathbf{2}$ & $1.49 \times 10^{5}$ & $4.77 \times 10^{3}$ & 0.9999 \\
$\mathbf{3}$ & $8.06 \times 10^{4}$ & $5.96 \times 10^{3}$ & 0.9993 \\
$\mathbf{4}$ & $6.34 \times 10^{4}$ & $6.45 \times 10^{3}$ & 0.9998 \\
$\mathbf{5}$ & $1.03 \times 10^{5}$ & $4.46 \times 10^{3}$ & 0.9993 \\
\hline
\end{tabular}

complex with TCNQ as evidenced by the appearance of absorption maxima at 743.0, 743.0, 742.0, 743.0 and $743.0 \mathrm{~nm}$ respectively (Table 1). The plot of (concentration of cyclophane) / absorbance (Y/A) vs $1 /$ concentration of guest (1/X) was linear. Benesi-Hildebrand equation was employed to calculate Ka values. ${ }^{26}$ From the slope and the intercept values, $K_{a}\left(K_{a}=\right.$ intercept $X$ 
slope $\left.^{-1}\right)$ and $\varepsilon\left(\varepsilon=\right.$ intercept $\left.^{-1}\right)$ were evaluated. The plot of (concentration of cyclophane) / absorbance (Y/A) vs $1 /$ concentration of guest $(1 / \mathrm{X})$ is linear suggesting that the predominate species in solution is a 1:1 complex (Fig. S3). The Ka, $\varepsilon$ and $r$ values of the CT complexes formed from 1, 1a, 1b, 2, 3, 4 and $\mathbf{5}$ with TCNQ are shown in Table 2. Benesi-Hildebrand treatment for the CT complex formed between the cyclophane amide 1a and TCNQ is shown in table 3 and Figure S3. CT spectra for cyclophane amide 1a and $\mathbf{2}$ with variable concentration of TCNQ are shown in Figure S1 and S2.

Table 3. Benesi-Hildebrand treatment data of the CT complex formed between the cyclophane amide, 1a and TCNQ

\begin{tabular}{ccc}
\hline Cyclophane amide & $\begin{array}{c}\lambda_{\max }(\mathrm{nm}) \text { of } \\
\text { cyclophane amide }\end{array}$ & $\begin{array}{c}\lambda_{\max }(\mathrm{nm}) \text { of TCNQ } \\
\text { complex }\end{array}$ \\
\hline $\mathbf{1}$ & 256.0 & 743.0 \\
$\mathbf{1 a}$ & 256.0 & 743.0 \\
$\mathbf{1 b}$ & 256.5 & 742.0 \\
$\mathbf{2}$ & 257.0 & 743.0 \\
$\mathbf{3}$ & 256.0 & 743.0 \\
$\mathbf{4}$ & 255.0 & 743.5 \\
$\mathbf{5}$ & 258.0 & 742.5 \\
\hline$\lambda_{\max }=743.5 \mathrm{~nm} ;$ concentration of cyclophane amide, $\mathbf{1 a}=1.89 \times 10^{-5} \mathrm{M}$ \\
$\mathrm{Ka}=6.65 \times 10^{4} \mathrm{M}^{-1} ; \varepsilon=6.87 \times 10^{3}\left[\mathrm{M}^{-1} \mathrm{~cm}^{-1}\right]$ and $\mathrm{r}=0.9998$.
\end{tabular}

\section{Conclusions}

In summary we have synthesized various cyclophane amides which show strong CT interactions selectively with TCNQ rather than TCNE and PQT. The biological activity and detailed charge transfer complexation studies of other similar cyclophane amides are under investigation.

\section{Experimental Section}

General. Melting points were determined by using a Toshniwal melting point apparatus by open capillary tube method and are uncorrected. The UV visible spectra were recorded on Shimadzu 2550 spectrophotometer. The IR spectra were performed on a PerkinElmer series 2000 FT-IR spectrophotometer. ${ }^{1} \mathrm{H}$ NMR and ${ }^{13} \mathrm{C}$ NMR spectra were recorded on a Bruker Advance 400 NMR spectrophotometer with TMS as internal standard. Mass spectra were determined by ESIMS using PerkinElmer Sciex, API 3000 spectrophotometer. Elemental analyses were performed on a Perkin-Elmer 240B elemental analyser. 


\section{General procedure for the synthesis of bis-oxy cyclophane amides (1-5)}

A solution of diamine $22(1.4 \mathrm{mmol})$ in dry dichloromethane $(200 \mathrm{~mL})$ and a solution of the corresponding diacid chloride 16, 16a, 16b, 17, 18, 19 and 20 (1.54 mmol) in dichloromethane (200 mL) were simultaneously added dropwise to a well stirred solution of triethylamine (3.1 $\mathrm{mmol})$ in dry dichloromethane $(400 \mathrm{~mL})$ for $8 \mathrm{~h}$. After the addition was complete the reaction mixture was stirred for another $24 \mathrm{~h}$. The solvent was removed at reduced pressure and the residue obtained was then dissolved in chloroform $(300 \mathrm{~mL})$, washed with water $(2 \times 200 \mathrm{~mL})$ to remove triethylammonium chloride and then dried over anhydrous sodium sulphate. Removal of the chloroform under reduced pressure gave the corresponding cyclophane amide as a crude material, which was purified by column chromatography $\left(\mathrm{SiO}_{2}\right)$.

Cyclophane amide (1). Yield 35\%, mp $315{ }^{\circ} \mathrm{C}$, IR $\left(\mathrm{KBr}, \mathrm{cm}^{-1}\right): 1713,1639,1600 .{ }^{1} \mathrm{H} \mathrm{NMR}$ $\left(400 \mathrm{MHz}, \mathrm{DMSO}-d_{6}\right) \delta 4.43(\mathrm{~d}, 4 \mathrm{H}, J=5.9 \mathrm{~Hz}), 5.24(\mathrm{~s}, 4 \mathrm{H}), 7.19-7.21(\mathrm{~m}, 2 \mathrm{H}), 7.38-7.46(\mathrm{~m}$, 13H), 7.49-7.52 (m, 3H), $7.56(\mathrm{~d}, 4 \mathrm{H}, J=8.0 \mathrm{~Hz}), 7.60(\mathrm{~d}, 2 \mathrm{H}, J=7.0 \mathrm{~Hz}), 9.06(\mathrm{t}, 2 \mathrm{H}, J=6$ $\mathrm{Hz}) .{ }^{13} \mathrm{C}$ NMR $\left(100 \mathrm{MHz}, \mathrm{DMSO}-d_{6}\right) \delta 42.7,67.2,113.2,118.7,119.54,124.4,127.2,128.0$, $128.2,128.9,129.4,129.5,135.1,136.5,139.2,139.6,141.3,158.7,166.4$. MS (ES) $m / z: 631.2$ $\left([\mathrm{M}+\mathrm{H}]^{+}\right)$. Anal. Calcd. for $\mathrm{C}_{42} \mathrm{H}_{34} \mathrm{~N}_{2} \mathrm{O}_{4}$ : C, 79.98; H, 5.43; N, 4.44\%. Found: C, 80.67; H, 5.47; $\mathrm{N}, 4.47 \%$.

Cyclophane amide (1a). Yield 40\%, mp $260{ }^{\circ} \mathrm{C}$, IR $\left(\mathrm{KBr}, \mathrm{cm}^{-1}\right): 1639,1583,1539 .{ }^{1} \mathrm{H}$ NMR $\left(400 \mathrm{MHz}, \mathrm{DMSO}-d_{6}\right) \delta 4.51(\mathrm{~d}, 4 \mathrm{H}, J=5.9 \mathrm{~Hz}), 5.13(\mathrm{~s}, 4 \mathrm{H}), 7.21-7.25(\mathrm{~m}, 2 \mathrm{H}), 7.41-7.44(\mathrm{~m}$, $10 \mathrm{H}), 7.46-7.58(\mathrm{~m}, 5 \mathrm{H}), 7.63(\mathrm{~d}, 1 \mathrm{H}, J=1.5 \mathrm{~Hz}), 7.67(\mathrm{~s}, 1 \mathrm{H}), 7.68(\mathrm{~d}, 4 \mathrm{H}, J=8.2 \mathrm{~Hz}), 7.73$ (bs, $1 \mathrm{H}), 9.03(\mathrm{t}, 2 \mathrm{H}, J=5.9 \mathrm{~Hz}) .{ }^{13} \mathrm{C} \mathrm{NMR}\left(100 \mathrm{MHz}, \mathrm{DMSO}-d_{6}\right) \delta 42.2,69.6,113.8,116.9$, 119.6, 119.9, 124.9, 127.1, 127.7, 128.2, 128.7, 129.1, 129.6, 136.7, 137.0, 138.8, 139.2, 140.9, 158.3, 166.8. MS (ES) $m / z: 631.2\left([\mathrm{M}+\mathrm{H}]^{+}\right)$. Anal. Calcd. for $\mathrm{C}_{42} \mathrm{H}_{34} \mathrm{~N}_{2} \mathrm{O}_{4}: \mathrm{C}, 79.98 ; \mathrm{H}, 5.43 ; \mathrm{N}$, 4.44\%. Found: C, 80.75; H, 5.46; N, 4.48\%.

Cyclophane amide (1b). Yield 52\%, mp $284{ }^{\circ} \mathrm{C}$, IR $\left(\mathrm{KBr}, \mathrm{cm}^{-1}\right): 1638,1583,1539 .{ }^{1} \mathrm{H}$ NMR $\left(400 \mathrm{MHz}, \mathrm{DMSO}-d_{6}\right) \delta 4.52(\mathrm{~d}, 4 \mathrm{H}, J=5.8 \mathrm{~Hz}), 5.19(\mathrm{~s}, 4 \mathrm{H}), 7.26-7.28(\mathrm{~m}, 2 \mathrm{H}), 7.39-7.45(\mathrm{~m}$, $10 \mathrm{H}), 7.48$ (s, 4H), 7.53 (d, 1H, $J=7.2 \mathrm{~Hz}$ ), 7.64 (d, 2H, J=7.5 Hz), 7.69 (d, 4H, J=8.1 Hz), $7.80(\mathrm{bs}, 1 \mathrm{H}), 9.01(\mathrm{t}, 2 \mathrm{H}, J=5.8 \mathrm{~Hz}) .{ }^{13} \mathrm{C}$ NMR (100 MHz, DMSO- $\left.d_{6}\right) \delta 42.0,68.9,114.8$, 116.2, 119.7, 125.0, 126.5, 127.1, 12.5, 128.1, 129.5, 129.7, 136.5, 138.7, 139.1, 140.8, 158.0, 166.5. MS (ES) $m / z: 631.2\left([\mathrm{M}+\mathrm{H}]^{+}\right)$. Anal. Calcd. for $\mathrm{C}_{42} \mathrm{H}_{34} \mathrm{~N}_{2} \mathrm{O}_{4}: \mathrm{C}, 79.98 ; \mathrm{H}, 5.43 ; \mathrm{N}, 4.44 \%$. Found: C, 80.55; H, 5.48; N, 4.47\%.

Cyclophane amide (2). Yield $42 \%, \mathrm{mp} 285{ }^{\circ} \mathrm{C}$, IR $\left(\mathrm{KBr}, \mathrm{cm}^{-1}\right): 1641,1584,1532 .{ }^{1} \mathrm{H}$ NMR $\left(400 \mathrm{MHz}, \mathrm{DMSO}-d_{6}\right) \delta 4.50(\mathrm{~d}, 4 \mathrm{H}, J=5.8 \mathrm{~Hz}), 5.30(\mathrm{~s}, 4 \mathrm{H}), 7.24-7.27(\mathrm{~m}, 2 \mathrm{H}), 7.40-7.45(\mathrm{~m}$, $10 \mathrm{H}), 7.55(\mathrm{t}, 1 \mathrm{H}, J=7.0 \mathrm{~Hz}), 7.63-7.67(\mathrm{~m}, 7 \mathrm{H}), 8.06(\mathrm{~s}, 1 \mathrm{H}), 8.39(\mathrm{~s}, 2 \mathrm{H}), 9.05(\mathrm{t}, 2 \mathrm{H}, J=6.0$ $\mathrm{Hz}$ ). MS (ES) $m / z: 676.4\left([\mathrm{M}+\mathrm{H}]^{+}\right)$. Anal. Calcd. for $\mathrm{C}_{42} \mathrm{H}_{33} \mathrm{~N}_{3} \mathrm{O}_{6}: \mathrm{C}, 74.65 ; \mathrm{H}, 4.92 ; \mathrm{N}, 6.22 \%$. Found: C, 74.86; H, 4.98; N, 6.31\%.

Cyclophane amide (3). Yield 43\%, mp $247{ }^{\circ} \mathrm{C}$, IR (KBr, $\left.\mathrm{cm}^{-1}\right): 1638,1588,1536 .{ }^{1} \mathrm{H}$ NMR $\left(400 \mathrm{MHz}, \mathrm{DMSO}-d_{6}\right) \delta 4.48,4.55$ (a pair of distorted doublet, $4 \mathrm{H}$ ), 5.15 (distorted ABq, $4 \mathrm{H}$ ), 7.23-7.26 (m, 5H), 7.34-7.54 (m, 11H), 7.65 (d, 2H, J=7.5 Hz), $7.71(\mathrm{~d}, 2 \mathrm{H}, J=7.3 \mathrm{~Hz}), 7.77$ 
(bs, 1H), 7.90-8.05 (m, 2H), $8.94(\mathrm{t}, 2 \mathrm{H}, J=8.5 \mathrm{~Hz})$. MS (ES) $m / z: 631.7\left([\mathrm{M}+\mathrm{H}]^{+}\right)$. Anal. Calcd. for $\mathrm{C}_{41} \mathrm{H}_{33} \mathrm{~N}_{3} \mathrm{O}_{4}$ : C, 77.95; H, 5.27; N, 6.65\%. Found: C, 78.16; H, 5.31; N, 6.69\%.

Cyclophane amide (4). Yield 30\%, mp $300{ }^{\circ} \mathrm{C}$, IR $\left(\mathrm{KBr}, \mathrm{cm}^{-1}\right): 1667,1602,1535 .{ }^{1} \mathrm{H}$ NMR $\left(400 \mathrm{MHz}, \mathrm{DMSO}-d_{6}\right) \delta 4.37$ (distorted doublet, $4 \mathrm{H}$ ), $4.56(\mathrm{~s}, 4 \mathrm{H}), 6.63(\mathrm{~d}, 3 \mathrm{H}, J=9.5 \mathrm{~Hz}$ ), 7.23 $(\mathrm{t}, 1 \mathrm{H}, J=7.6 \mathrm{~Hz}), 7.30(\mathrm{~d}, 4 \mathrm{H}, J=7.2 \mathrm{~Hz}), 7.49(\mathrm{~d}, 1 \mathrm{H}, J=7.0 \mathrm{~Hz}) 7.58(\mathrm{~d}, 2 \mathrm{H}, J=6.9 \mathrm{~Hz})$ $7.65(\mathrm{~d}, 4 \mathrm{H}, J=7.4 \mathrm{~Hz}) 7.82(\mathrm{~s}, 1 \mathrm{H}), 8.67$ (distorted triplet, 2H). MS (ES) $\mathrm{m} / \mathrm{z}: 496.7$ $\left(\left[\mathrm{M}+\mathrm{NH}_{4}\right]^{+}\right)$. Anal. Calcd. for $\mathrm{C}_{30} \mathrm{H}_{26} \mathrm{~N}_{2} \mathrm{O}_{4}: \mathrm{C}, 75.30 ; \mathrm{H}, 5.48 ; \mathrm{N}, 5.85 \%$. Found: C, 75.59; H, $5.53 ; \mathrm{N}, 5.89 \%$.

Cyclophane amide (5). Yield 40\%, mp $260{ }^{\circ} \mathrm{C}$, IR $\left(\mathrm{KBr}, \mathrm{cm}^{-1}\right): 1742,1660,1634,1531 .{ }^{1} \mathrm{H}$ NMR (400 MHz, DMSO- $\left.d_{6}\right) \delta 4.31(\mathrm{~d}, 4 \mathrm{H}, J=6.1 \mathrm{~Hz}), 4.55(\mathrm{~s}, 4 \mathrm{H}), 6.49,6.51$ (dd, 2H, $J=2.2$ $\mathrm{Hz}), 7.09(\mathrm{~d}, 2 \mathrm{H}, J=2.0 \mathrm{~Hz}), 7.13(\mathrm{~s}, 1 \mathrm{H}), 7.21(\mathrm{~d}, 2 \mathrm{H}, J=9.0 \mathrm{~Hz}), 7.43(\mathrm{ABq}, 8 \mathrm{H}, J=8.0 \mathrm{~Hz})$, $7.55-7.64(\mathrm{~m}, 3 \mathrm{H}), 8.85(\mathrm{t}, 2 \mathrm{H}, J=6.1 \mathrm{~Hz}) .{ }^{13} \mathrm{C} \mathrm{NMR}\left(100 \mathrm{MHz}, \mathrm{DMSO}-d_{6}\right) \delta .42 .2,67.5,109.7$, 112.3, 124.1, 124.7, 127.0, 128.5, 128.6, 128.9, 129.5, 135.0, 138.9, 139.5, 141.3, 156.2, 168.2. MS (ES) $m / z: 529.2\left([\mathrm{M}+\mathrm{H}]^{+}\right)$. Anal. Calcd. for $\mathrm{C}_{34} \mathrm{H}_{28} \mathrm{~N}_{2} \mathrm{O}_{4}: \mathrm{C}, 77.25 ; \mathrm{H}, 5.34 ; \mathrm{N}, 5.30 \%$. Found: C, 77.48; H, 5.41; N, 5.38\%.

CT complexation studies of cyclophane amides 1, 1a, 1b, 2, 3, 4 and 5 with TCNQ. A solution of TCNQ $\left(4.9 \times 10^{-6} \mathrm{M}\right)$ in $\mathrm{CH}_{3} \mathrm{CN}$ at various dilutions $(1 \mathrm{~mL}, 2 \mathrm{~mL}, 3 \mathrm{~mL}, 4 \mathrm{~mL}, 5 \mathrm{~mL}$ and $6 \mathrm{~mL}$ ) were prepared and added to the solution of the cyclophane amide 1, 1a, 1b, 2, 3, 4 and $5\left(1.89 \times 10^{-5} \mathrm{M}\right)$ in a $1: 1$ mixture of $\mathrm{CHCl}_{3} / \mathrm{CH}_{3} \mathrm{CN}(3 \mathrm{~mL})$ in a quartz cuvette of path length 1 $\mathrm{cm}$. The UV-Vis spectrum was also obtained for each of the sample separately and the changes in the absorbance of CT bands were recorded.

\section{Supplementary Material}

CT spectra of cyclophane amides 1a and 2 (Figure S1 and S2) and plot between 1/X and Y/A for cyclophane amide 1a (Figure S3) are available.

\section{Acknowledgements}

The authors thank DST, New Delhi for financial assistance, RSIC, CDRI, Lucknow for FAB MS, and DST-FIST for providing NMR facility for the Department. RP thanks Orchid Chemicals \& Pharmaceuticals Ltd., for providing the laboratory and analytical facility.

\section{References}

1. Krzysztof, E. K.; Jerald, S.B.; Daria, J. Z. Chem. Rev. 1989, 89, 929-972. 
2. (a) Ulrich, L. Liebigs Ann. Chem. 1987, 11, 949-955. (b) Dirk, L.; Rainer, A. J. B.; Helmar, G. Liebigs Ann. 1994, 1, 199-201.

3. (a) Jingsong, Y.; Xiaoqi, Y.; Changlu, L.; Rugang, X. Synth. Commun. 1999, 29, 2447-2455.

(b) Yukito, M.; Junichi, K.; Hiroaki, T. J. Chem. Soc. Chem. Commun. 1985, 753-755.

4. Thomas, R. W.; Yuan, F.; Cynthia, J. B. J. Org. Chem. 1989, 54, 1584-1589.

5. Hosseini, M. W.; Lehn, J. M.; Du, S. R.; Gu, K.; Mertes, M. P. J. Org. Chem. 1987, 52, 1662-1666.

6. Lehn, J. M. Science (Washington, D.C.). Inclusion Compounds 1985, 227, 849-859.

7. Yohannes, P. G.; Mertes, M. P.; Mertes, K. B. J. Am. Chem. Soc. 1985, 107, 8288-8289.

8. Groves, J. T.; Stern, M. K. J. Am. Chem. Soc. 1988, 110, 8628-8638.

9. Groves, J. T.; Lee, J.; Marla, S. S. J. Am. Chem. Soc. 1997, 119, 6269-6273.

10. Lehn, J. M. Supramolecular chemistry: Concepts and Perspectives VCH: Weinheim, Germany, 1995.

11. Costero, A. M,; Banuls, M. J.; Aurell, M. J.; Ward, M. D.; Argent, S. Tetrahedron 2004, 60, 9471-9478.

12. (a) Szumna, A.; Jurczak, J. A. Eur. J. Org. Chem. 2001, 21, 4031-4039. (b) Chmielewski, M.; Jurczak, J. Tetrahedron Lett. 2004, 45, 6007-6010.

13. Christopher, A. H.; Duncan, H. P. Angew. Chem. Int. Ed. Engl. 1992, 31, 792-795.

14. Kyu, C. S.; Donna, V. E.; Erkang, F.; Andreq, D. H. J. Am. Chem. Soc. 1991, 113, 76407645.

15. Claire, M. K.; David, A. L. Angew. Chem. Int. Ed. 2004, 43, 1222-1223.

16. Jhaumeer-Laulloo, B. S. Asian J. Chem. 2000, 12, 775-780.

17. Subodh, K.; Vandana, B.; Harijit, S. Tetrahedron 1998, 54, 5575-5586.

18. Yehia, A.; Ahmed, H. M. Synthesis 1993, 5, 503-508.

19. Rajkumar.P.; Abdul Rasheed, A. M.; Balu. P. M.; Murugesan. K. Bioorg. Med. Chem. 2006, 14, 7458-7467.

20. Rajkumar.P.; Abdul Rasheed, A. M.; Rabia. A. I.; Chamundeeswari. D. Bioorg. Med. Chem. Lett. 2006, 16, 6019-6023.

21. Bauer, H.; Stier, F.; Petry, C.; Stadler, C.; Staab, H. A. Eur J. Org. Chem. 2001, 3255-3279.

22. Sorenson, A. P.; Nielsen. M. B.; Becher, J. Tetrahedron Lett. 2003, 44, 2979-2982.

23. (a) Rajakumar, P.; Selvam, S. Tetrahedron 2007, 63, 8891-8901; (b) Rajakumar, P.; Padmanabhan, R. Tetrahedron Lett. 2010, 51, 1059-1063. .

24. Hart, H.; Rajakumar, P. Tetrahedron 1995, 51, 1313-1336.

25. (a) Blazevic, N.; Kolbah, D.; Belin, B.; Sunjic, V.; Kajfez, F. Synthesis 1979, 161-165. (b) Bottini, A. T.; Vasu Dev.; Klinck, J. Organic Syntheses 1963, 43, 6-9. (c) Meyers, A. I.; Warmus, J. S.; Dilley, G. J. Organic Syntheses 1996, 73, 246-249. (d) Rajakumar, P.; Padmanabhan, R. Tetrahedron 2011, 67, 9669-9679.

26. Benesi, H. A.; Hildebrand, J. H. J. Am. Chem. Soc. 1949, 71, 2703-2707. 